\title{
Highly Sensitive Textile-Based Capacitive Pressure Sensors Using PVDF-HFP/Ionic Liquid Composite Films
}

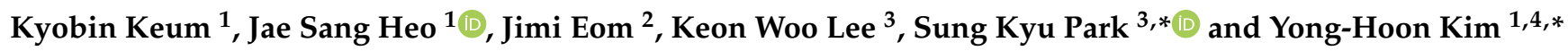 \\ 1 School of Advanced Materials Science and Engineering, Sungkyunkwan University, Suwon 16419, Korea; \\ fiete1202@skku.edu (K.K.); heojs38@gmail.com (J.S.H.) \\ 2 Advanced Textile R\&D Department, Research Institute of Convergence Technology, Korea Institute of \\ Industrial Technology (KITECH), Ansan 15588, Korea; jimi0321@naver.com \\ 3 School of Electrical and Electronic Engineering, Chung-Ang University, Seoul 06974, Korea; \\ 1kw9941@gmail.com \\ 4 SKKU Advanced Institute of Nanotechnology (SAINT), Sungkyunkwan University, Suwon 16419, Korea \\ * Correspondence: skpark@cau.ac.kr (S.K.P.); yhkim76@skku.edu (Y.-H.K.)
}

Citation: Keum, K.; Heo, J.S.; Eom, J. Lee, K.W.; Park, S.K.; Kim, Y.-H. Highly Sensitive Textile-Based Capacitive Pressure Sensors Using PVDF-HFP/Ionic Liquid Composite Films. Sensors 2021, 21, 442. https:// doi.org/10.3390/s21020442

Received: 30 November 2020 Accepted: 8 January 2021 Published: 9 January 2021

Publisher's Note: MDPI stays neutral with regard to jurisdictional clai$\mathrm{ms}$ in published maps and institutional affiliations.

Copyright: (C) 2021 by the authors. Licensee MDPI, Basel, Switzerland. This article is an open access article distributed under the terms and conditions of the Creative Commons Attribution (CC BY) license (https:// creativecommons.org/licenses/by/ $4.0 /)$.

\begin{abstract}
Textile-based pressure sensors have garnered considerable interest in electronic textiles due to their diverse applications, including human-machine interface and healthcare monitoring systems. We studied a textile-based capacitive pressure sensor array using a poly(vinylidene fluoride)-cohexafluoropropylene (PVDF-HFP)/ionic liquid (IL) composite film. By constructing a capacitor structure with Ag-plated conductive fiber electrodes that are embedded in fabrics, a capacitive pressure sensor showing high sensitivity, good operation stability, and a wide sensing range could be created. By optimizing the PVDF-HFP:IL ratio (6.5:3.5), the fabricated textile pressure sensors showed sensitivity of $9.51 \mathrm{kPa}^{-1}$ and $0.69 \mathrm{kPa}^{-1}$ in the pressure ranges of $0-20 \mathrm{kPa}$ and $20-100 \mathrm{kPa}$, respectively. The pressure-dependent capacitance variation in our device was explained based on the change in the contact-area formed between the multi-filament fiber electrodes and the PVDF-HFP/IL film. To demonstrate the applicability and scalability of the sensor device, a $3 \times 3$ pressure sensor array was fabricated. Due to its matrix-type array structure and capacitive sensing mechanism, multi-point detection was possible, and the different positions and the weights of the objects could be identified.
\end{abstract}

Keywords: electronic textiles; capacitive pressure sensors; PVDF-HFP; ionic liquid; contact area

\section{Introduction}

Electronic textiles (e-textiles) have recently garnered considerable interest in the field of wearable electronics due to the diverse applications that are enabled by integrating them into various sensor devices [1-3], triboelectric nanogenerators [4-6], wireless communication devices [7-9], logic circuits [10], and control systems [11]. Unlike conventional electronic devices, e-textiles can be made virtually unnoticeable by fully embedding them in clothes or fabrics. Therefore, applications requiring an 'always-on' mode operation can be more effectively performed by using e-textiles. Among various electronic components that can be integrated into e-textiles are strain sensors [12,13], motion sensors [14], environmental biosensors [15,16], and pressure sensors $[17,18]$. In particular, the integration of pressure sensors has been widely investigated to broaden the applications of e-textiles in breath monitoring [19], heart monitoring [20], and human-machine interface systems [21-23]. In these applications, the required pressure sensitivity and pressuredetection ranges are varied. For example, in breath and heart monitoring, pressure sensors should have a high sensitivity in the low-pressure range [24]; while in applications such as body-weight monitoring, sensors should have higher detection range and better sensitivity in the high-pressure range [25]. From this perspective, pressure sensors that possess both high sensitivity and a wide sensing range are in high demand in e-textile applications. 
There are several types of mechanisms for pressure detection. These include resistive, piezoresistive, piezoelectric, and capacitive types [26]. The capacitive-type pressure-sensing mechanism can especially provide advantages such as multi-point detection and low power consumption [11,27-29]. Considering that e-textiles typically require low-power operation due to the limitation of their power supply, capacitive-type pressure sensors can be a good candidate for e-textiles. For the realization of textile-based capacitive pressure sensors, various device structures and materials have been investigated. For example, Li et al. demonstrated a capacitive pressure sensor based on a honeycomb-weaving architecture that exhibited a sensitivity of $0.045 \mathrm{kPa}^{-1}$ under $10 \mathrm{kPa}$ [30]. Vu et al. studied a capacitive pressure sensor using polyester/spandex (PET/SP) fabrics with a combination of singlewalled carbon nanotubes and stretchable silver ink [25]. The fabricated sensor exhibited a sensitivity of $0.02-0.042 \mathrm{kPa}^{-1}$ depending on the number of encapsulation lines. In another approach, Choi et al. studied a porous elastomer/multiwalled carbon nanotube composite as a sensing material that exhibited a pressure sensitivity of $6.42 \mathrm{kPa}^{-1}$ and $1.72 \mathrm{kPa}^{-1}$ in the range of $0-2 \mathrm{kPa}$ and $2-10 \mathrm{kPa}$, respectively [31]. Wu et al. utilized silver nanofibercoated conductive fabrics and a spacer fabric to construct a textile pressure sensor [32] that exhibited a sensitivity of $0.283 \mathrm{kPa}^{-1}$ and good operational stability. Although these previous investigations are noteworthy, the pressure sensitivities were relatively low, and the detection ranges were somewhat limited. Among various material candidates for capacitive pressure sensors, ionic liquid (IL)-based soft ion-gel films are of significant interest due to their high dielectric constants. Ion-gel films have been frequently adopted as a gate dielectric layer in thin-film transistors to reduce the operation voltage and enhance the electrical properties [33-35]. In capacitive pressure sensors, the high-k ion-gel film can also enhance their response to the applied pressure and their sensitivity [36]. In addition, ion-gel films possess good mechanical flexibility and stability, which could enable their integration in e-textile devices.

In this study, we demonstrate a textile-based capacitive pressure sensor using a poly(vinylidene fluoride)-co-hexafluoropropylene (PVDF-HFP)/IL composite film. The PVDF-HFP/IL film is used as a dielectric layer in the capacitor, positioned between two fabric sheets with multi-filament Ag-plated fiber electrodes. The Ag-plated fibers and the PVDF-HFP/IL film formed a cross-point capacitor structure in which its capacitance value varies with the applied pressure. The fabricated capacitive pressure sensor exhibited high sensitivity, good stability, and wide sensing range. Particularly, with optimized PVDFHFP:IL ratio (6.5:3.5), the sensor showed a sensitivity of $9.51 \mathrm{kPa}^{-1}$ and $0.69 \mathrm{kPa}^{-1}$ in the pressure ranges of $0-20 \mathrm{kPa}$ and $20-100 \mathrm{kPa}$, respectively. The response times of the sensor under loading and unloading conditions were $-0.8 \mathrm{~s}$ and $-0.5 \mathrm{~s}$, respectively. The pressure-dependent capacitance variation is explained based on the change in the contact area, which is formed between the multi-filament fiber electrodes and the PVDF-HFP/IL film. To demonstrate the applicability and scalability of the sensor device, we used a $3 \times 3$ pressure sensor array. Due to its matrix-type array structure and the capacitive sensing mechanism, multi-point detection was possible, and it successfully identified the different positions and weights of the objects.

\section{Materials and Methods}

The fabrication process of PVDF-HFP/IL film is shown in Figure 1a. To fabricate the PVDF-HFP/IL film, a drop-casting method was used. To prepare the PVDF-HFP solution, PVDF-HFP pellets (average $\mathrm{M}_{\mathrm{w}}-455,000$, Sigma Aldrich, St. Louis, MO, USA) were dissolved in acetone at $10 \mathrm{wt} \%$ and thoroughly stirred for $2 \mathrm{~h}$. Then, the PVDF-HFP solution and 1-ethyl-3-methylimidazolium bis(trifluoromethylsulfonyl)imide ([EMIM][TFSI]) IL $\left(\geq 98 \%, \mathrm{M}_{\mathrm{w}}=391.31\right.$, purchased from Sigma Aldrich) were mixed at different weight ratios and stirred for $-1 \mathrm{~h}$. The weight ratios of PVDF-HFP:IL varied as 5:5, 6:4, and 6.5:3.5. To fabricate a freestanding PVDF-HFP/IL film, which is used as a dielectric layer in the textile pressure sensor, a $3 \mathrm{~cm} \times 3 \mathrm{~cm}$ bare glass substrate was prepared and thoroughly cleaned with acetone, isopropanol alcohol, and deionized water, then dried with dry ni- 
trogen. The PVDF-HFP/IL solution was dropped on the cleaned glass substrate using a micropipette with a dropping volume of $-10 \mu \mathrm{L}$. Next, to cure the film, a thermal annealing at $40{ }^{\circ} \mathrm{C}$ was carried out for $12 \mathrm{~h}$. After the curing, the film was detached from the glass substrate. The average diameter of the fabricated PVDF-HFP/IL film was $-0.5 \mathrm{~cm}$. To fabricate the pressure sensor, Ag-plated conductive nylon fibers (fiber diameter: 280d, SOITEX, Goyang, Korea) was stitched on both the top and bottom polyester fabric sheets, which had dimensions of $5 \mathrm{~cm} \times 5 \mathrm{~cm}$. A double-sided thermoplastic adhesive film (Thermal Bonding Film 583, 3M, Maplewood, MN, USA) was then bonded to the bottom polyester fabric sheet by applying $2.56 \mathrm{kPa}$ of pressure at $110^{\circ} \mathrm{C}$ for $10 \mathrm{~min}$. To expose the Ag-plated fiber electrode, which was located at the center region of the fabric sheet, the center region of the adhesive film was cut to a dimension of $-0.5 \mathrm{~cm} \times 0.5 \mathrm{~cm}$. Then, the free-standing PVDF-HFP/IL film was placed on top of the Ag-plated fiber electrode. Next, a top fabric sheet having Ag-plated fiber electrode was covered, and finally the sample was hot pressed for $1 \mathrm{~min}$ by applying $2.56 \mathrm{kPa}$ of pressure at $60^{\circ} \mathrm{C}$ to bond the fabric sheets together.

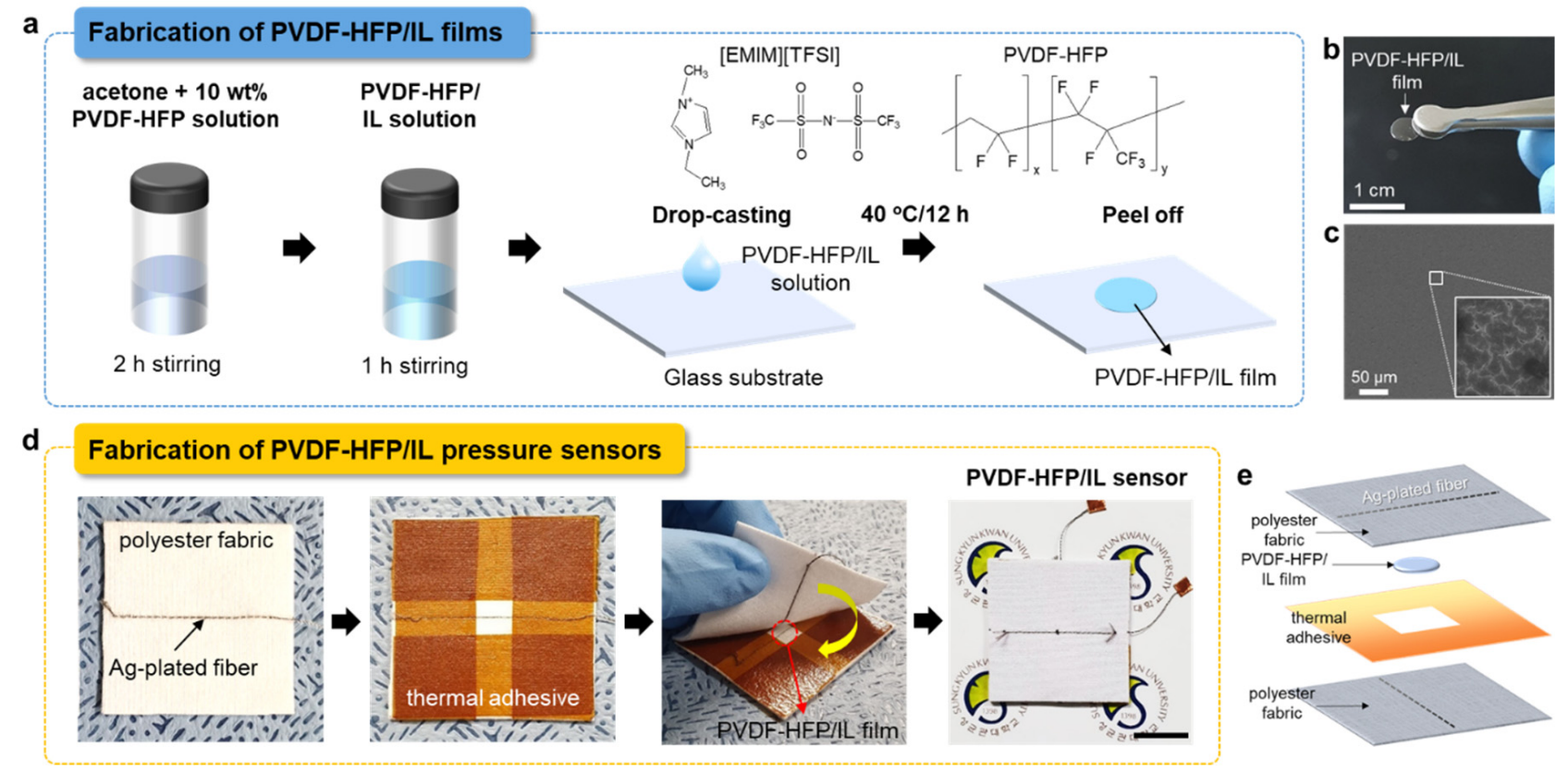

Figure 1. (a) The fabrication process of the PVDF-HFP/IL film using the drop-casting method and the molecular structures of [EMIM][TFSI] and PVDF-HFP. (b) An optical image, and (c) an FESEM image of PVDF-HFP/IL film (PVDF-HFP:IL = 6.5:3.5). (d) The fabrication process of PVDF-HFP/IL-based textile pressure sensor (scale bar: $2 \mathrm{~cm}$ ). (e) A schematic structure of the PVDF-HFP/IL-based textile pressure sensor.

The surface morphology of the PVDF-HFP/IL film was analyzed using a fieldemission scanning electron microscope (FESEM) (JSM-7600F, JEOL, Tokyo, Japan). To measure the pressure-sensing characteristics, a force gauge (M5-2, MARK-10, Copiague, NY, USA) was utilized. The measured pressure range was $0-100 \mathrm{kPa}$. The characterizations of the pressure sensor were carried out using a probe station (model 8000, MS-TECH, Hwaseong, Korea) connected to an LCR meter (4284A, Agilent Technologies, Inc., Santa Clara, CA, USA) and a semiconductor parameter analyzer (4200A-SCS, Keithley, Cleveland, $\mathrm{OH}, \mathrm{USA})$. The capacitance value of the pressure sensor was measured with an applied voltage of $1 \mathrm{~V}$ at frequencies of $0.1-100 \mathrm{kHz}$.

\section{Results}

\subsection{The Device Structure and Electrical Characterization of PVDF-HFP/IL Films}

The optical and FESEM images of the PVDF-HFP/IL film are shown in Figure 1b,c, respectively (PVDF-HFP:IL ratio of 6.5:3.5). As shown in Figure 1c, the PVDF-HFP:IL film had a granular-like morphology, showing small micro-cracks on the film surface. Using 
the PVDF-HFP:IL film, a textile-based capacitive pressure sensor was fabricated following the procedure described in Figure 1d. Figure 1e shows the schematic structure of the pressure sensor. To form a capacitor structure, the PVDF-HFP/IL film was sandwiched between top and bottom fabric sheets with multi-filament Ag-plated conducting fibers. The electrical resistance of the Ag-plated conducting fiber was about $1 \Omega / \mathrm{cm}$, and the top and bottom Ag-plated fibers were positioned orthogonally to form a cross-point structure. To firmly attach the two fabric sheets, a thermal adhesive film was inserted between the fabric sheets and hot-pressed. As described, the PVDF-HFP/IL film played important roles in our sensor device, as a dielectric layer for capacitor formation and as a basis for capacitance variation under applied pressure. The PVDF-HFP/IL film was composed of PVDF-HFP and IL; the PVDF-HFP formed a network structure, and the ILs contributed to the formation of the electric double layer (EDL). Among various available polymer materials, PVDF-HFP was selected as the matrix polymer due to its good mechanical stability and easy film-forming ability due to fluorination and its low surface energy [37,38]. PVDF-HFP has good thermal stability, good electrochemical stability, low crystallinity, and high dielectric constant $(\varepsilon=8.4)$ [39]; therefore, the composite of PVDF-HFP and IL with high ionic conductivity could be effective as a sensing material in capacitive-type pressure sensors. Under an applied bias, the IL molecules migrated and redistributed near the electrodes and formed EDLs. Since the amount of ILs in the PVDF-HFP/IL film had a strong influence on the initial capacitance value and the pressure sensitivity of the sensor, it was necessary to optimize the ratio of PVDF-HFP and IL in the film. In our study, the PVDF-HFP:IL ratio was varied as 6.5:3.5, 6:4, and 5:5. When the IL ratio was too high (typically higher than 5), a gel-like film was obtained instead of a dense free-standing film, due to the lack of PVDF-HFP component in the film. As a consequence, detaching the film from the glass substrate and handling the film were difficult. However, when the IL ratio was lower than 3.5, the viscosity of the solution was considerably increased, and when the film was cured, an abnormal cone shape structure was formed at the center region of the film, possibly due to the high viscosity of the PVDF-HFP/IL solution. This could prevent the formation of uniform contacts between the Ag-plated fiber electrodes and the PVDF-HFP/IL film. Therefore, in our experiment, the PVDF-HFP:IL ratio was set in the range of 6.5:3.5-5:5.

To investigate the effects of PVDF-HFP:IL ratio on the initial capacitance $\left(C_{0}\right)$ value of the film, PVDF-HFP/IL films with different ratios were prepared. Figure 2a shows the change of $C_{0}$ as a function of PVDF-HFP:IL ratio. When the PVDF-HFP:IL ratio was varied as 6.5:3.5, 6:4 and 5:5, the $\mathrm{C}_{0}$ increased to $0.062 \mathrm{nF}, 0.85 \mathrm{nF}$ and $9.32 \mathrm{nF}$, respectively. The increased $\mathrm{C}_{0}$ can be attributed to the larger amount of ILs in the PVDF-HFP/IL film, which contributed to the EDL formation at the interfaces with the top and bottom fiber electrodes. According to the Stern theory [40], the ions of opposite polarity to the electrode move toward the electrode, forming a Stern layer near the interface. In addition, the ions that had the same polarity as the electrode moved away from the interface and diffused into the film to form a diffusion layer [40]. Due to the formation of EDLs at both electrodes that had an opposite polarity, a high capacitance was induced. One of the unique characteristics of an IL-based dielectric layer is a large frequency-dependent capacitance variation [36,41] due to the relatively slow migration of ILs compared to that of dipole polarization occurring in typical inorganic dielectric films $[36,41]$. Figure $2 b$ shows the change of initial capacitance value as a function of the frequency. As displayed, the $\mathrm{C}_{0}$ value decreased from $-25 \mathrm{nF}$ to $0.062 \mathrm{nF}$ when the frequency was increased from $100 \mathrm{~Hz}$ to $100 \mathrm{kHz}$. In our study, because the initial capacitance value directly affected the pressure sensitivity (S) as shown in the following equation:

$$
\mathrm{S}=\frac{\Delta \mathrm{C} / \mathrm{C}_{0}}{\Delta \mathrm{P}}=\frac{\left(\mathrm{C}-\mathrm{C}_{0}\right) / \mathrm{C}_{0}}{\mathrm{P}-\mathrm{P}_{0}}
$$

where $\mathrm{P}$ is the applied pressure, optimization of the operation frequency was necessary. Additionally, the mechanical stability of PVDF-HFP/IL film is important in achieving highly stable devices. To evaluate the mechanical stability of PVDF-HFP/IL film, we 
performed a cyclic bending test of the pressure sensor ( 500 cycles at a bending radius of $2.5 \mathrm{~mm}$ ) and traced the relative change of the $C_{0}$ value. As shown in Figure $2 c$, the $C_{0}$ value was only changed by $2.7 \%$ after the bending test, indicating that the PVDF-HFP/IL film had good mechanical stability.
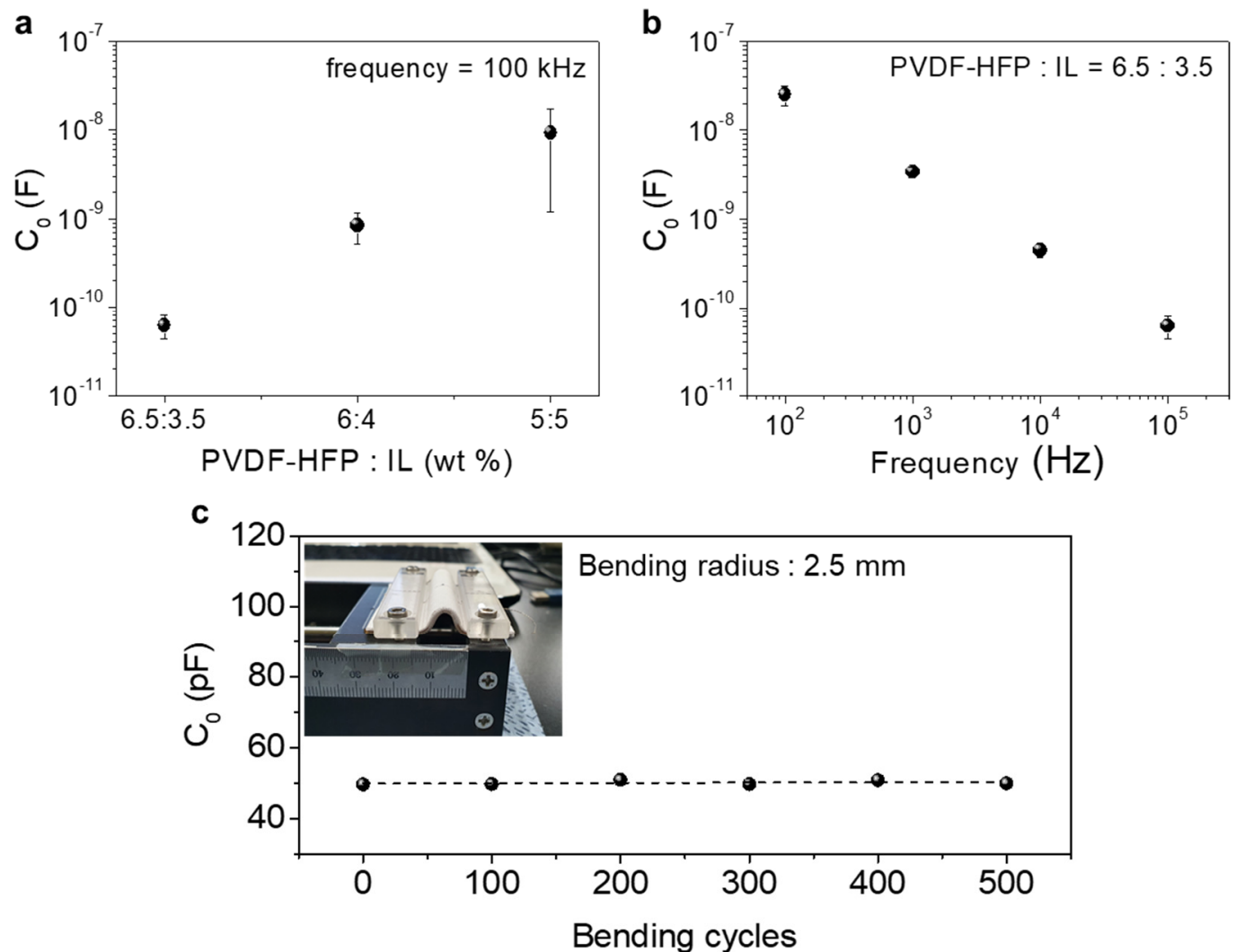

Figure 2. (a) The variation of initial capacitance as a function of the PVDF-HFP:IL ratio (frequency $=100 \mathrm{kHz}$ ). (b) The variation of initial capacitance as a function of the frequency (PVDF-HFP:IL = 6.5:3.5). (c) The initial capacitance $\left(\mathrm{C}_{0}\right)$ variation of the PVDF-HFP/IL textile pressure sensor during the cyclic bending test (500 bending cycles, bending radius of $2.5 \mathrm{~mm}$ ). The inset shows an optical image of the textile pressure sensor under bent condition.

\subsection{Pressure-Sensing Characteristics of PVDF-HFP/IL-Based Textile Pressure Sensors}

To examine the effects of the PVDF-HFP:IL ratio on sensing characteristics such as pressure sensitivity and detection range, textile-based pressure sensors using PVDFHFP/IL films with different PVDF-HFP:IL ratios were fabricated. Figure 3a shows the plots of $\Delta \mathrm{C} / \mathrm{C}_{0}$ vs. P measured from each pressure sensor at a frequency of $100 \mathrm{kHz}$. The capacitance variation $\left(\Delta \mathrm{C} / \mathrm{C}_{0}\right)$ was largely dependent on the PVDF-HFP:IL ratio, and particularly, the sensors fabricated with PVDF-HFP:IL ratio of 6.5:3.5 exhibited the highest capacitance change, while those fabricated with PVDF-HFP:IL ratios of 5:5 and $6: 4$ showed much reduced capacitance variations (Figure $3 b$ ). Figure $3 c$ summarizes the pressure sensitivities obtained in the ranges of $0-20 \mathrm{kPa}\left(\mathrm{S}_{1}\right)$ and $20-100 \mathrm{kPa}\left(\mathrm{S}_{2}\right)$. When the PVDF-HFP:IL ratio was $6.5: 3.5$, the $S_{1}$ and $S_{2}$ were $9.51 \mathrm{kPa}^{-1}$ and $0.69 \mathrm{kPa}^{-1}$, respectively. However, as the IL weight ratio was increased, the $S_{1}$ and $S_{2}$ decreased substantially. One of the main reasons for the reduced sensitivity was the high $C_{0}$ values of the film (Figure 2a). Specifically, the $\mathrm{C}_{0}$ value increased by one to two orders of magnitude when the PVDF-HFP:IL ratio was changed from 6.5:3.5 to 6:4 and 5:5. In all samples, there was a considerable transition of pressure sensitivity at around $\mathrm{P}-20 \mathrm{kPa}$. It was expected that at this pressure value, the dominant mechanism for the capacitance variation would change. In the low-pressure range, the variation of the contact area that formed between the 
multi-filament fiber electrodes and the PVDF-HFP/IL film was dominant, and induced a large capacitance change. In contrast, in the high-pressure range, the contact-area variation became less dominant, and the structural deformation of the PVDF-HFP/IL film became more dominant. Based on these results, we claim that the sensor fabricated with PVDFHFP:IL ratio of 6.5:3.5 had a wider detection range and a higher sensitivity.
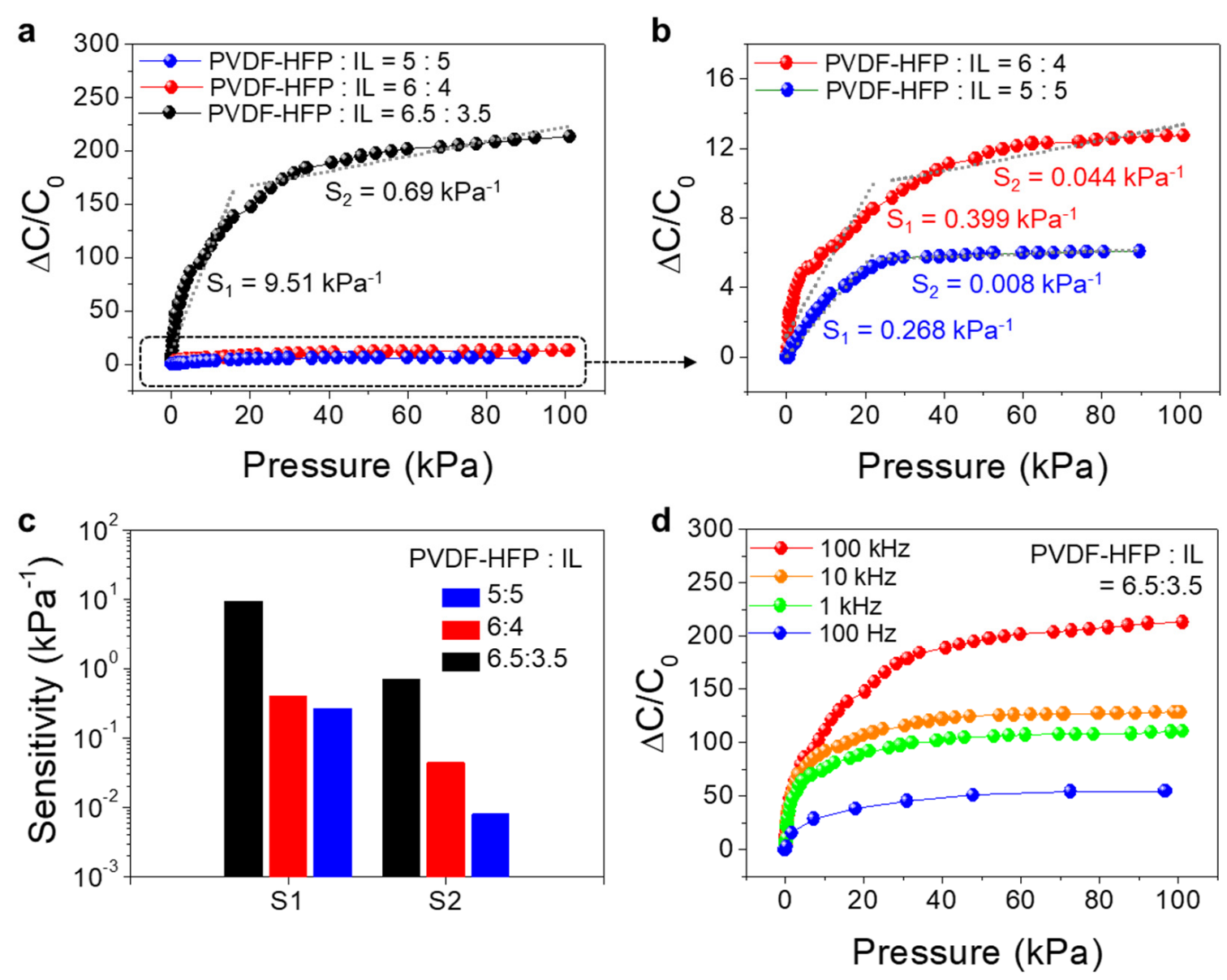

Figure 3. $(\mathbf{a}, \mathbf{b})$ Pressure-sensing characteristics $\left(\Delta C / C_{0}\right.$ vs. $\left.P\right)$ of PVDF-HFP/IL-based pressure sensors fabricated with different PVDF-HFP:IL ratios (5:5 (blue), 6:4 (red), and 6.5:3.5 (black). (c) The pressure sensitivities in the low-pressure $(0-20 \mathrm{kPa})\left(S_{1}\right)$ and high-pressure $(20-100 \mathrm{kPa})$ regions. $S_{1}$ and $S_{2}$ represent the sensitivity values at low pressure and high pressure, respectively. (d) Frequency-dependent pressure-sensing characteristics of the PVDF-HFP/IL-based pressure sensors (PVDF-HFP:IL ratio $=6.5: 3.5$ ).

Additionally, because the capacitance of PVDF-HFP/IL film is also varied by the frequency, the frequency-dependent pressure-sensing characteristics were evaluated. As shown in Figure 3d, as the frequency decreased from $100 \mathrm{kHz}$ to $0.1 \mathrm{kHz}$, the capacitance variation $\left(\Delta C / C_{0}\right)$ by the applied pressure decreased, which can be also attributed to the higher $C_{0}$ values at lower frequencies (Figure 2b). More importantly, the response at high pressure $\left(\mathrm{S}_{2}\right)$ was relatively low when the frequency was $0.1-10 \mathrm{kHz}$, limiting the detection range of the sensor. In overall, the results suggest that a PVDF-HFP:IL ratio of 6.5:3.5 and an operation frequency of $100 \mathrm{kHz}$ are the most suitable to achieve high-pressure sensitivity and a wider pressure-detection range. 


\subsection{Pressure-Sensing Mechanism}

In the PVDF-HFP/IL textile pressure sensor, the two multi-filament Ag-plated fiber electrodes and the PVDF-HFP/IL dielectric film formed a capacitor. Therefore, the capacitance can be expressed by the following equation:

$$
C=\varepsilon \frac{A}{d}
$$

where $\varepsilon$ is permittivity of the dielectric, $A$ is the area, and $d$ is the thickness of the dielectric film. Then, by applying an external pressure, the area and the thickness of the dielectric film can be changed, thereby increasing the capacitance. In our device structure, it was suggested that although the thickness of PVDF-HFP/IL film was decreased by pressure, the increase of contact area made between the multi-filament fiber electrodes and the PVDFHFP/IL film was considered as the dominant mechanism for the capacitance variation [36], particularly at low pressures. When there was no external force, the contact area made between the fiber electrodes and the PVDF-HFP/IL film was small where the EDL can be formed (Figure 4a). However, as the applied pressure was increased, the multi-filament fiber structure was deformed and pushed into the PVDF-HFP/IL film, which increased the contact area of the EDL formation (Figure $4 \mathrm{~b}$ ). As a result, the capacitance value increased with the applied pressure. However, after a certain level of pressure, in our case at around $\mathrm{P}-20 \mathrm{kPa}$, the rate of contact-area increase was reduced, and the capacitance variation was also reduced, resulting in lower sensitivity (Figure $3 \mathrm{a}, \mathrm{b}$ ).

a

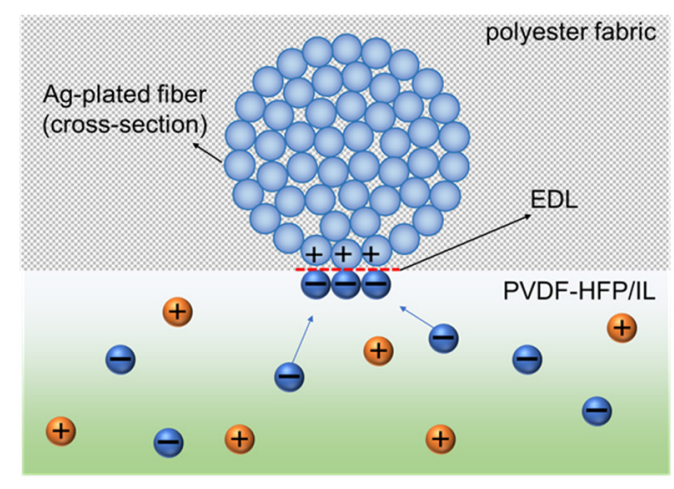

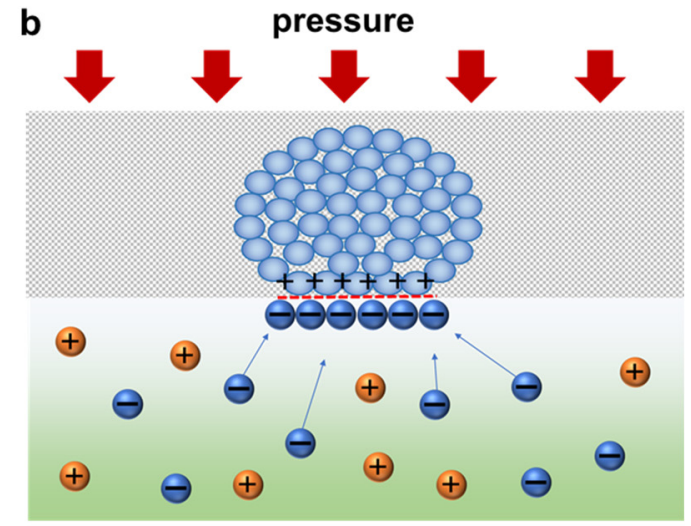

Figure 4. Pressure-sensing mechanism of the PVDF-HFP/IL-based pressure sensor. (a) Without an external pressure, the contact area that formed between the fiber electrode and the PVDF-HFP/IL film was relatively small, resulting in lower EDL formation. (b) With an external pressure, the contact area and the EDL formation region were extended, increasing the capacitance value.

\subsection{Response Time and the Stability of PVDF-HFP/IL Pressure Sensors}

The response characteristics of the pressure sensor are also important parameters for determining the applications of the sensor. Figure 5a shows the response characteristics of PVDF-HFP/IL textile pressure sensor (PVDF-HFP:IL ratio = 6.5:3.5). Under the loading condition, the extracted response time was $-0.8 \mathrm{~s}$, and under the unloading condition, the response time was $-0.5 \mathrm{~s}$. The response rate of our sensor was relatively slow compared to previous IL-based pressure sensors or fabric-based pressure sensors (0.1-0.3 s) [42-45]. The relatively slow response characteristics were due to the viscoelastic property of the PVDF-HFP/IL film and the complicated weaving structure of the multifilament fiber electrodes on the fabric sheet $[36,46-48]$. However, considering the potential applications of PVDF-HFP/IL pressure sensors, such as monitoring of pressure distribution from a body, these response characteristics could be sufficient $[36,48]$. Additionally, the operation stability of the PVDF-HFP/IL pressure sensor was evaluated. Figure $5 b$ shows the results from a cyclic test carried out for 1300 cycles of loading and unloading. As displayed, the sensor 
exhibited relatively stable operation up to 1300 cycles without noticeable degradation of the sensing performance. It should be noted that in the initial period of the cyclic test, the capacitance value in unloading states increased slightly and later stabilized. This can be attributed to the formation of a closer contact between the fiber electrodes and the PVDF-HFP/IL film due to repeated pressure.
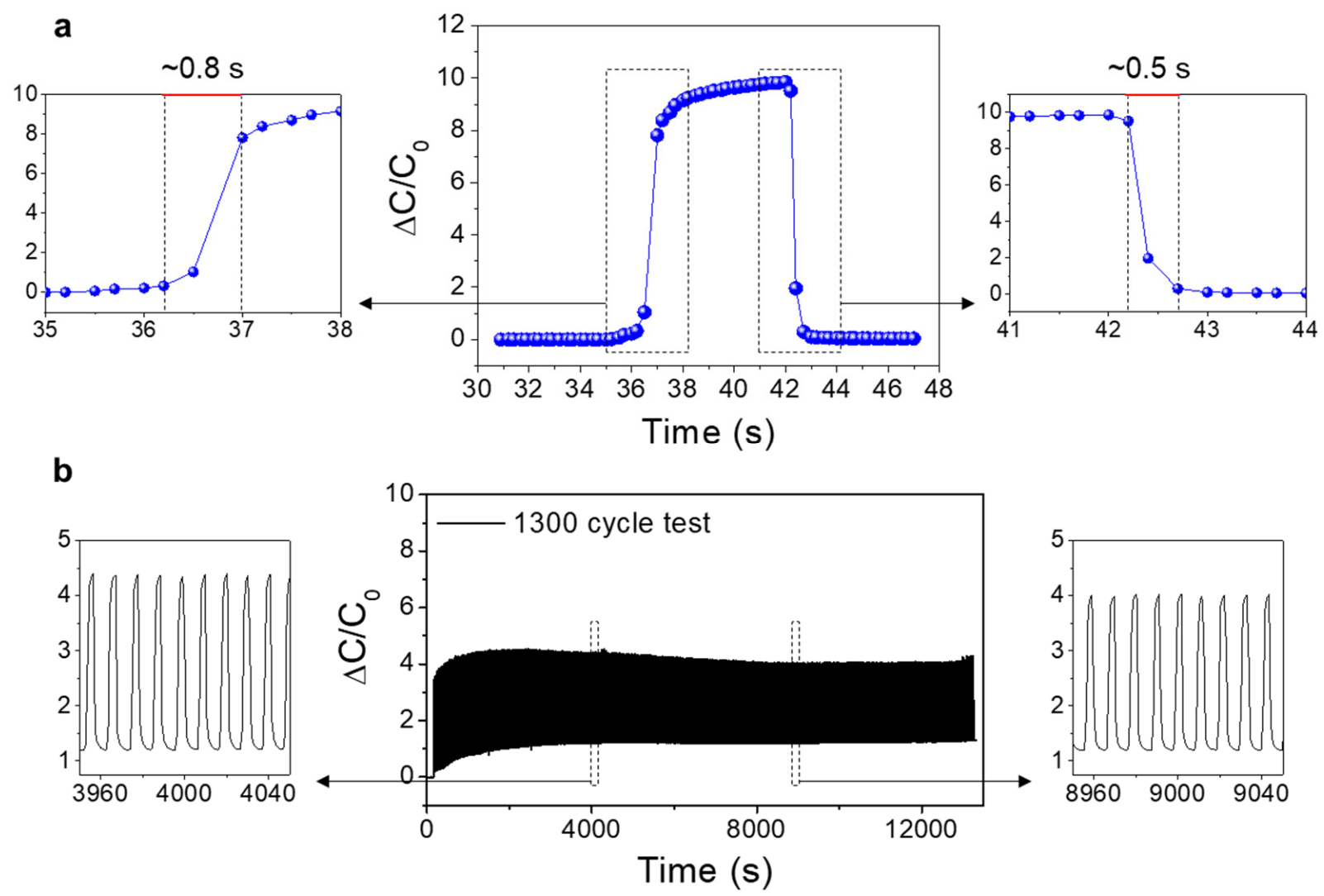

Figure 5. (a) The response characteristics of the PVDF-HFP/IL textile pressure sensor (PVDF-HFP:IL ratio = 6.5:3.5, loading pressure $=2 \mathrm{kPa}$ ). The response times for loading and unloading conditions were $-0.8 \mathrm{~s}$ and $-0.5 \mathrm{~s}$, respectively.

(b) The cyclic test results for the PVDF-HFP/IL textile pressure sensor (1300 cycles, loading pressure $=1 \mathrm{kPa})$.

\subsection{Multi-Point Pressure Detection Using a $3 \times 3$ Pressure Sensor Array}

To demonstrate the applicability of the PVDF-HFP/IL pressure sensors, a matrix-type $3 \times 3$ pressure sensor array was fabricated using polyester fabrics. Figure $6 a, b$ show the schematic layout and optical image of the $3 \times 3$ pressure sensor array. Since the PVDFHFP/IL-based pressure sensor had a cross-point structure, a matrix-type array could be simply constructed for multi-point detection. The column fiber electrodes were designated as $X_{n}(n=1,2,3)$, and the row fiber electrodes were designated as $Y_{n}(n=1,2,3)$ (Figure 6a). As shown in Figure $6 \mathrm{~b}$, each sensing point was designated as $P_{n}(n=1-9)$. Because the $3 \times 3$ pressure sensor array operated based on the capacitance variation, a multi-point pressure detection was possible. To demonstrate the multi-point pressure detection, one to three separate objects were placed at different sensing points on the sensor array (Figure $6 c-f$ ). The mapping of the capacitance values was carried out by using a homemade array tester composed of an LCR meter and a digital multimeter/switch system [36]. As shown in Figure $6 \mathrm{~g}-\mathrm{j}$, the $3 \times 3$ sensor array could detect the positions and the weights of the objects without significant crosstalk between the sensing points. These results suggest that the textile pressure sensors implementing PVDF-HFP/IL film and multi-filament fiber electrodes could be successfully adopted in e-textile applications for multi-point detection. 
a

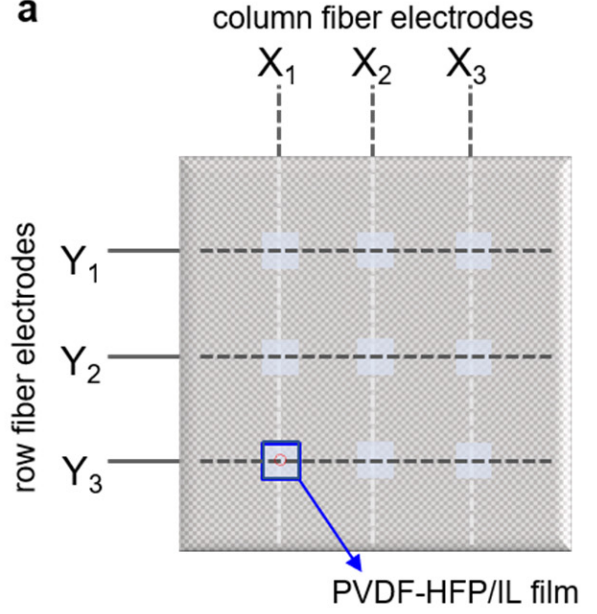

C

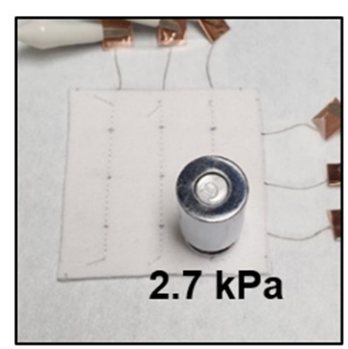

g

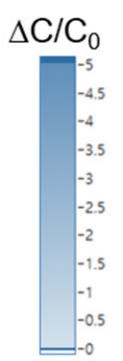

d

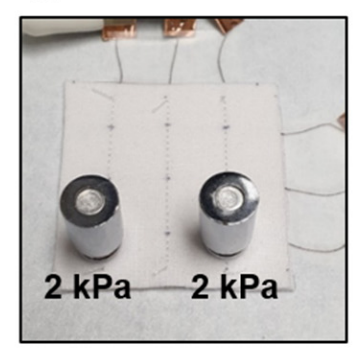

h

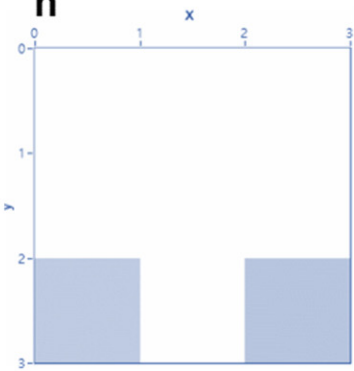

b

\section{$3 \times 3$ pressure sensor array}
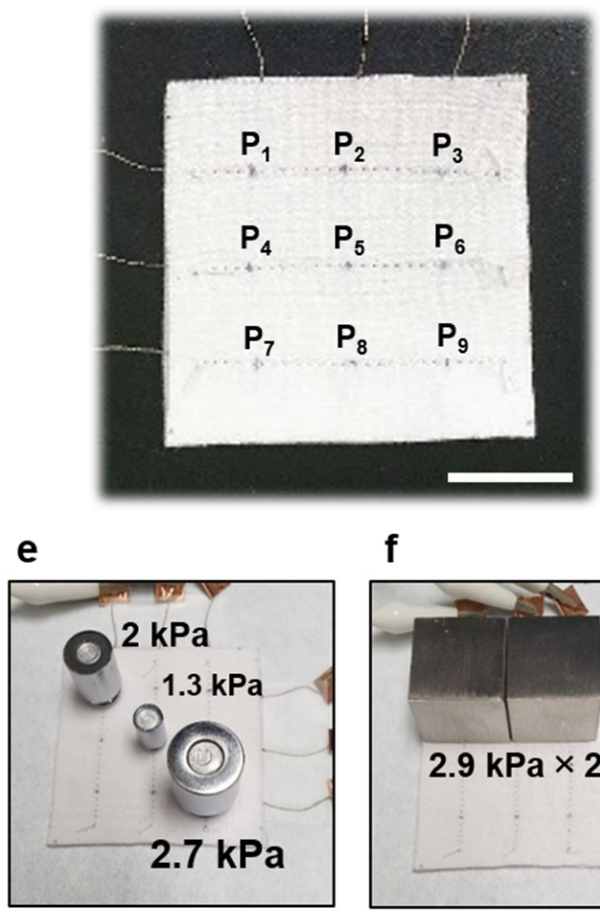

f
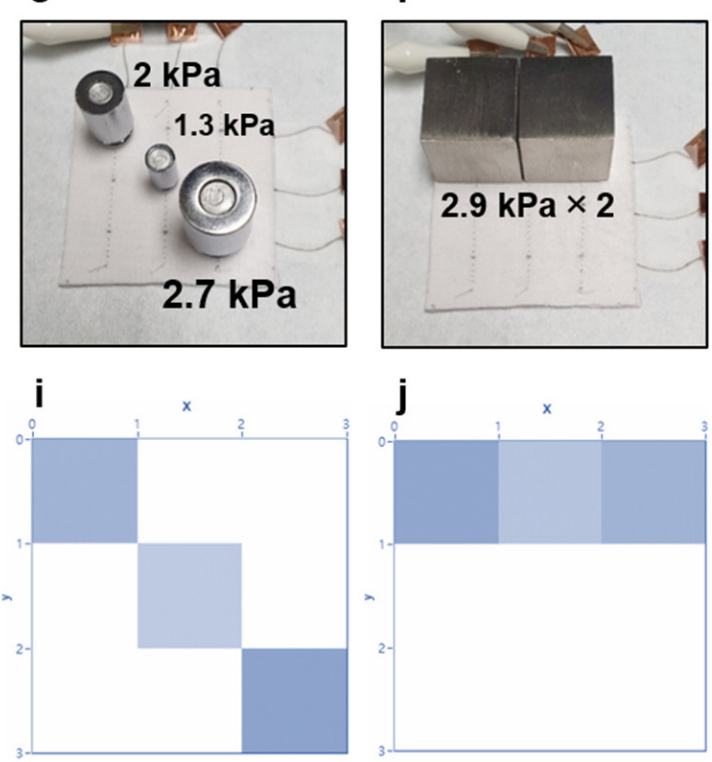

j

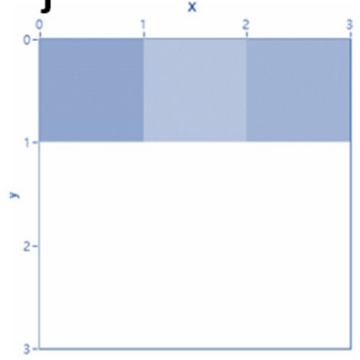

Figure 6. (a) The schematic layout, and (b) an optical image of a $3 \times 3$ PVDF-HFP/IL-based textile pressure sensor array. $X_{1}, X_{2}$, and $X_{3}$ represent the column fiber electrodes, and $Y_{1}, Y_{2}$, and $Y_{3}$ represent the row fiber electrodes (scale bar: $2 \mathrm{~cm}$ ). (c-f) Optical images of the pressure sensor array loaded with various weights. (g-j) Corresponding pressure mapping data obtained from the sensor array.

\section{Conclusions}

In our study, we demonstrated a textile-based capacitive pressure sensor using a PVDFHFP/IL composite film and multi-filament Ag-plated fiber electrodes. Using an optimized PVDF-HFP:IL ratio, we created a textile pressure sensor that exhibited a high pressure sensitivity, a wide detection range, and good operation stability. The PVDF-HFP:IL ratio had significant effects on the sensitivity and detection range of the sensor. The pressuredependent capacitance variation was explained based on the change in the contact area, which was formed between the multi-filament fiber electrodes and the PVDF-HFP/IL film. To demonstrate the applicability and scalability of the PVDF-HFP/IL-based sensor, we used a $3 \times 3$ pressure sensor array; due to its matrix-type array structure and the capacitive-type sensing mechanism, identification of the different positions and weights of the objects was possible. 
Author Contributions: Methodology, analysis, investigation, writing—original draft preparation: K.K.; methodology, analysis, writing —review and editing: J.S.H., J.E.; data curation: K.W.L.; supervision: S.K.P., Y.-H.K. All authors have read and agreed to the published version of the manuscript.

Funding: This research was partially supported by the Chung-Ang University Research Scholarship Grants in 2019, and by the Korea Institute of Industrial Technology as "Development of smart textronic products based on electronic fibers and textiles (KITECH JA-20-0001)".

Conflicts of Interest: The authors declare no conflict of interest.

\section{References}

1. Nie, B.; Huang, R.; Yao, T.; Zhang, Y.; Miao, Y.; Liu, C.; Liu, J.; Chen, X. Textile-Based Wireless Pressure Sensor Array for Human-Interactive Sensing. Adv. Funct. Mater. 2019, 29, 1808786. [CrossRef]

2. Wang, C.; Li, X.; Gao, E.; Jian, M.; Xia, K.; Wang, Q.; Xu, Z.; Ren, T.; Zhang, Y. Carbonized Silk Fabric for Ultrastretchable, Highly Sensitive, and Wearable Strain Sensors. Adv. Mater. 2016, 28, 6640-6648. [CrossRef] [PubMed]

3. Wu, R.; Ma, L.; Hou, C.; Meng, Z.; Guo, W.; Yu, W.; Yu, R.; Hu, F.; Liu, X.Y. Silk Composite Electronic Textile Sensor for High Space Precision 2D Combo Temperature-Pressure Sensing. Small 2019, 15, 1901558. [CrossRef] [PubMed]

4. Xiong, J.; Cui, P.; Chen, X.; Wang, J.; Parida, K.; Lin, M.-F.; Lee, P.S. Skin-touch-actuated textile-based triboelectric nanogenerator with black phosphorus for durable biomechanical energy harvesting. Nat. Commun. 2018, 9, 4280. [CrossRef] [PubMed]

5. Jao, Y.-T.; Yang, P.-K.; Chiu, C.-M.; Lin, Y.-J.; Chen, S.-W.; Choi, D.; Lin, Z.-H. A textile-based triboelectric nanogenerator with humidity-resistant output characteristic and its applications in self-powered healthcare sensors. Nano Energy 2018, 50, 513-520. [CrossRef]

6. Seung, W.; Gupta, M.K.; Lee, K.Y.; Shin, K.-S.; Lee, J.-H.; Kim, T.Y.; Kim, S.; Lin, J.; Kim, J.H.; Kim, S.-W. Nanopatterned Textile-Based Wearable Triboelectric Nanogenerator. ACS Nano 2015, 9, 3501-3509. [CrossRef]

7. Tian, X.; Lee, P.M.; Tan, Y.J.; Wu, T.L.Y.; Yao, H.; Zhang, M.; Li, Z.; Ng, K.A.; Tee, B.C.K.; Ho, J.S. Wireless body sensor networks based on metamaterial textiles. Nat. Electron. 2019, 2, 243-251. [CrossRef]

8. Lee, T.; Lee, W.; Kim, S.-W.; Kim, J.J.; Kim, B.-S. Flexible Textile Strain Wireless Sensor Functionalized with Hybrid Carbon Nanomaterials Supported ZnO Nanowires with Controlled Aspect Ratio. Adv. Funct. Mater. 2016, 26, 6206-6214. [CrossRef]

9. Loss, C.; Gonçalves, R.; Lopes, C.; Pinho, P.; Salvado, R. Smart Coat with a Fully-Embedded Textile Antenna for IoT Applications. Sensors 2016, 16, 938. [CrossRef]

10. Bae, H.; Jang, B.C.; Park, H.; Jung, S.-H.; Lee, H.M.; Park, J.-Y.; Jeon, S.-B.; Son, G.; Tcho, I.-W.; Yu, K.; et al. Functional Circuitry on Commercial Fabric via Textile-Compatible Nanoscale Film Coating Process for Fibertronics. Nano Lett. 2017, 17, 6443-6452. [CrossRef]

11. Lee, J.; Kwon, H.; Seo, J.; Shin, S.; Koo, J.H.; Pang, C.; Son, S.; Kim, J.H.; Jang, Y.H.; Kim, D.H.; et al. Conductive Fiber-Based Ultrasensitive Textile Pressure Sensor for Wearable Electronics. Adv. Mater. 2015, 27, 2433-2439. [CrossRef] [PubMed]

12. Eom, J.; Heo, J.-S.; Kim, M.; Lee, J.H.; Park, S.K.; Kim, Y.-H. Highly sensitive textile-based strain sensors using poly(3,4ethylenedioxythiophene):polystyrene sulfonate/silver nanowire-coated nylon threads with poly-L-lysine surface modification. RSC Adv. 2017, 7, 53373-53378. [CrossRef]

13. Luo, C.; Tian, B.; Liu, Q.; Feng, Y.; Wu, W. One-Step-Printed, Highly Sensitive, Textile-Based, Tunable Performance Strain Sensors for Human Motion Detection. Adv. Mater. Technol. 2020, 5, 1900925. [CrossRef]

14. Eom, J.; Jaisutti, R.; Lee, H.; Lee, W.; Heo, J.-S.; Lee, J.-Y.; Park, S.K.; Kim, Y.-H. Highly Sensitive Textile Strain Sensors and Wireless User-Interface Devices Using All-Polymeric Conducting Fibers. ACS Appl. Mater. Interfaces 2017, 9, 10190-10197. [CrossRef] [PubMed]

15. Jeerapan, I.; Sempionatto, J.R.; Pavinatto, A.; You, J.-M.; Wang, J. Stretchable biofuel cells as wearable textile-based self-powered sensors. J. Mater. Chem. A 2016, 4, 18342-18353. [CrossRef]

16. Mishra, R.K.; Martín, A.; Nakagawa, T.; Barfidokht, A.; Lu, X.; Sempionattom, J.R.; Lyu, K.M.; Karajic, A.; Musameh, M.M.; Kyratzis, I.L.; et al. Detection of vapor-phase organophosphate threats using wearable conformable integrated epidermal and textile wireless biosensor systems. Biosens. Bioelectron. 2018, 101, 227-234. [CrossRef]

17. Atalay, O.; Atalay, A.; Gafford, J.; Walsh, C. A Highly Sensitive Capacitive-Based Soft Pressure Sensor Based on a Conductive Fabric and a Microporous Dielectric Layer. Adv. Mater. Technol. 2018, 3, 1700237. [CrossRef]

18. Li, R.; Si, Y.; Zhu, Z.; Guo, Y.; Zhang, Y.; Pan, N.; Sun, G.; Pan, T. Supercapacitive Iontronic Nanofabric Sensing. Adv. Mater. 2017, 1700253. [CrossRef]

19. Ma, L.; Wu, R.; Patil, A.; Zhu, S.; Meng, Z.; Meng, H.; Hou, C.; Zhang, Y.; Liu, Q.; Yu, R.; et al. Full-Textile Wireless Flexible Humidity Sensor for Human Physiological Monitoring. Adv. Funct. Mater. 2019, 29, 1904549. [CrossRef]

20. Koo, H.R.; Lee, Y.-J.; Gi, S.; Khang, S.; Lee, J.H.; Lee, J.-H.; Lim, M.-G.; Park, H.-J.; Lee, J.-W. The Effect of Textile-Based Inductive Coil Sensor Positions for Heart Rate Monitoring. J. Med. Syst. 2014, 38, 2. [CrossRef]

21. Liu, M.; Pu, X.; Jiang, C.; Liu, T.; Huang, X.; Chen, L.; Du, C.; Sun, J.; Hu, W.; Wang, Z.L. Large-Area All-Textile Pressure Sensors for Monitoring Human Motion and Physiological Signals. Adv. Mater. 2017, 29, 1703700. [CrossRef] [PubMed] 
22. Cao, R.; Pu, X.; Du, X.; Yang, W.; Wang, J.; Guo, H.; Zhao, S.; Yuan, Z.; Zhang, C.; Li, C.; et al. Screen-Printed Washable Electronic Textiles as Self-Powered Touch/Gesture Tribo-Sensors for Intelligent Human-Machine Interaction. ACS Nano 2018, 12, $5190-5196$. [CrossRef] [PubMed]

23. Zhou, Z.; Li, Y.; Cheng, J.; Chen, S.; Hu, R.; Yan, X.; Liao, X.; Xu, C.; Yu, J.; Li, L. Supersensitive all-fabric pressure sensors using printed textile electrode arrays for human motion monitoring and human-machine interaction. J. Mater. Chem. C 2018, 6, 13120. [CrossRef]

24. Chen, S.; Wu, N.; Ma, L.; Lin, S.; Yuan, F.; Xu, Z.; Li, W.; Wang, B.; Zhou, J. Noncontact Heartbeat and Respiration Monitoring Based on a Hollow Microstructured Self-Powered Pressure Sensor. ACS Appl. Mater. Interfaces 2018, 10, 3660-3667. [CrossRef]

25. Vu, C.C.; Kim, J. Highly elastic capacitive pressure sensor based on smart textiles for full-range human motion monitoring Sens. Actuator A Phys. 2020, 314, 112029. [CrossRef]

26. Jeon, S.; Lim, S.-C.; Trung, T.Q.; Jung, M.; Lee, N.-E. Flexible Multimodal Sensors for Electronic Skin: Principle, Materials, Device, Array Architecture, and Data Acquisition Method. Proc. IEEE 2019, 107, 2065-2083. [CrossRef]

27. Dobrzynska, J.A.; Gijs, M.A.M. Flexible Polyimide-Based Force Sensor. Sens. Actuator A Phys. 2012, 173, 127-135. [CrossRef]

28. Nie, B.; Li, R.; Cao, J.; Brandt, J.D.; Pan, T. Flexible Transparent Iontronic Film for Interfacial Capacitive Pressure Sensing. Adv. Mater. 2015, 27, 6055-6062. [CrossRef]

29. Baxter, L.K. Capacitive Sensors: Design and Applications; IEEE Press: New York, NY, USA, 1997.

30. Li, S.; Li, R.; Chen, T.; Xiao, X. Highly Sensitive and Flexible Capacitive Pressure Sensor Enhanced by Weaving of Pyramidal Concavities Staggered in Honeycomb Matrix. IEEE Sens. J. 2020, 20, 14436-14443. [CrossRef]

31. Choi, J.; Kwon, D.; Kim, K.; Park, J.; Orbe, D.D.; Gu, J.; Ahn, J.; Cho, I.; Jeong, Y.; Oh, Y.; et al. Synergetic Effect of Porous Elastomer and Percolation of Carbon Nanotube Filler toward High Performance Capacitive Pressure Sensors. ACS Appl. Mater. Interfaces 2020, 12, 1698-1706. [CrossRef]

32. Wu, R.; Ma, L.; Patil, A.; Hou, C.; Zhu, S.; Fan, X.; Lin, H.; Yu, W.; Guo, W.; Liu, X.Y. All-Textile Electronic Skin Enabled by Highly Elastic Spacer Fabric and Conductive Fibers. ACS Appl. Mater. Interfaces 2019, 11, 33336-33346. [CrossRef] [PubMed]

33. Cho, J.H.; Lee, J.; Xia, Y.; Kim, B.; He, Y.; Renn, M.J.; Lodge, T.P.; Frisbie, C.D. Printable ion-gel gate dielectrics for low-voltage polymer thin-film transistors on plastic. Nat. Mater. 2008, 7, 900-906. [CrossRef] [PubMed]

34. Pu, J.; Yomogida, Y.; Liu, K.-K.; Li, L.-J.; Iwasa, Y.; Takenobu, T. Highly Flexible MoS2 Thin-Film Transistors with Ion Gel Dielectrics. Nano Lett. 2012, 12, 4013-4017. [CrossRef] [PubMed]

35. Lee, K.H.; Kang, M.S.; Zhang, S.; Gu, Y.; Lodge, T.P.; Frisbie, C.D. "Cut and Stick” Rubbery Ion Gels as High Capacitance Gate Dielectrics. Adv. Mater. 2012, 24, 4457-4462. [CrossRef] [PubMed]

36. Keum, K.; Eom, J.; Lee, J.H.; Heo, J.S.; Park, S.K.; Kim, Y.-H. Fully-integrated wearable pressure sensor array enabled by highly sensitive textile-based capacitive ionotronic devices. Nano Energy 2021, 79, 105479. [CrossRef]

37. Chhetry, A.; Kim, J.; Yoon, H.; Park, J.Y. Ultrasensitive Interfacial Capacitive Pressure Sensor Based on a Randomly Distributed Microstructured Iontronic Film for Wearable Applications. ACS Appl. Mater. Interfaces 2019, 11, 3438-3449. [CrossRef]

38. Lu, J.; Yan, F.; Texter, J. Advanced applications of ionic liquids in polymer science. Prog. Polym. Sci. 2009, 34, 431-448. [CrossRef]

39. Song, S.; Wang, J.; Tang, J.; Muchakayala, R.; Ma, R. Preparation, properties, and Li-ion battery application of EC + PC-modified PVdF-HFP gel polymer electrolyte films. Ionics 2017, 23, 3365-3375. [CrossRef]

40. Ueno, K.; Shimotani, H.; Yuan, H.; Ye, J.; Kawasaki, M.; Iwasa, Y. Field-Induced Superconductivity in Electric Double Layer Transistors. J. Phys. Soc. Jpn. 2014, 83, 032001. [CrossRef]

41. Lee, S.W.; Shin, M.; Park, J.Y.; Kim, B.S.; Tu, D.; Jeon, S.; Jeong, U. Thin Ion-Gel Dielectric Layer to Enhance the Stability of Polymer Transistors. Sci. Adv. Mater. 2015, 7, 874-880. [CrossRef]

42. Yang, X.; Wang, Y.; Sun, H.; Qing, X. A flexible ionic liquid-polyurethane sponge capacitive pressure sensor. Sens. Actuator A Phys. 2019, 285, 67-72. [CrossRef]

43. Zhang, L.; Li, H.; Lai, X.; Gao, T.; Yang, J.; Zeng, X. Thiolated Graphene@Polyester Fabric-Based Multilayer Piezoresistive Pressure Sensors for Detecting Human Motion. ACS Appl. Mater. Interfaces 2018, 10, 41784-41792. [CrossRef] [PubMed]

44. Ho, D.H.; Cheon, S.; Hong, P.; Park, J.H.; Suk, J.W.; Kim, D.H.; Han, J.T.; Cho, J.H. Multifunctional Smart Textronics with Blow-Spun Nonwoven Fabrics. Adv. Funct. Mater. 2019, 29, 1900025. [CrossRef]

45. Zhang, Q.; Wang, Y.L.; Xia, Y.; Zhang, P.F.; Kirk, T.V.; Chen, X.D. Textile-Only Capacitive Sensors for Facile Fabric Integration without Compromise of Wearability. Adv. Mater. Technol. 2019, 4, 1900485. [CrossRef]

46. Yin, M.-J.; Zhang, Y.; Yin, Z.; Zheng, Q.; Zhang, A.P. Micropatterned Elastic Gold-Nanowire/Polyacrylamide Composite Hydrogels for Wearable Pressure Sensors. Adv. Mater. Technol. 2018, 3, 1800051. [CrossRef]

47. Mao, Y.; Lin, S.; Zhao, X.; Anand, L. A large deformation viscoelastic model for double-network hydrogels. J. Mech. Phys. Solids 2017, 100, 103-130. [CrossRef]

48. Yin, M.-J.; Yin, Z.; Zhang, Y.; Zheng, Q.; Zhang, A.P. Micropatterned elastic ionic polyacrylamide hydrogel for low-voltage capacitive and organic thin-film transistor pressure sensors. Nano Energy 2019, 58, 96-104. [CrossRef] 